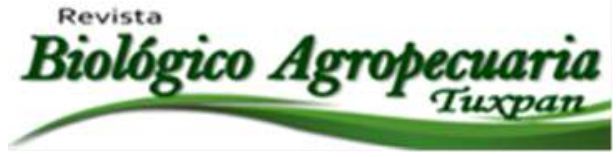

\title{
Proyecto de inversión de aguacate booth 8 en la huasteca veracruzana, una oportunidad de negocio para los productores como generador de divisas
}

Booth 8 avocado investment project in the huasteca veracruzana, a business opportunity for producers as a generator of foreign currency

\author{
Mendo Muñoz, Rubén de la Paz ${ }^{1 凶}$; Cruz Ramírez, Benjamín ${ }^{1}$; González Salas, Miguel Ángel ${ }^{1}$ \\ y Gutiérrez Vivanco, Jordán ${ }^{1}$. \\ ${ }^{1}$ Universidad Veracruzana. Facultad de Ciencias Biológicas y agropecuarias campus Tuxpan \\ ${ }^{\square}$ Autor para correspondencia: $\underline{\text { trre_mendo@ hotmail.com }}$
}

Recibido: $15 / 07 / 2017$

Aceptado: 10/12/2017

\section{RESUMEN}

La industria del aguacate en México, en los últimos años se ha incrementado notablemente, actualmente somos el primer país productor de aguacate, el fruto que es producido en nuestro país es destinado a mercados internacionales de gran valor como Estados Unidos, principal importador de aguacate que se produce en México, el aguacate es muy demandado tanto como en el mercado local, así como el internacional (SAGARPA, 2011). En este proyecto de inversión sobre la producción, comercialización y exportación del aguacate BOOTH 8 con las características de estudios como; mercado, técnico, organizacional y económico -financiero se dará a conocer un diagnostico situacional actual del aguacate en México y el mercado meta que será Estados Unidos, al cual se pretende exportar dicho fruto de la mejor manera. El proyecto de inversión para la producción, comercialización y exportación de aguacate de la variedad Booth 8, nos muestra que se cuenta con la información necesaria para producir, comercializar y exportarlo además se precisó el mercado meta donde arrojo que la parte del pacifico es donde hay más consumo del aguacate y que además prefieren de variedades verdes como lo son el Booth 8 y el Hass. Estados Unidos es un mercado amplio con más de 300 millones de habitantes casi el $45 \%$ consume el fruto del aguacate. El precio que se manejara en el mercado local será $\$ 15$ por pieza y en el mercado meta elevara su costo a $\$ 35$ por lo que concluimos que se puede decir que el proyecto de producción, comercialización y exportación de aguacate Booth 8 hacia el mercado meta, es factible y rentable.

Palabras clave: Aguacate, Seguridad alimentaria, comercialización.

\begin{abstract}
ABSTRAC
The avocado industry in Mexico, in recent years has increased significantly, we are currently the first producer of avocado, the fruit that is produced in our country is destined to high-value international markets such as the United States, the main avocado importer that It is produced in Mexico, the avocado
\end{abstract}


is very demanded as much as in the local market, as well as the international one (SAGARPA, 2011). In this investment project on the production, marketing and export of BOOTH 8 avocado with the characteristics of studies such as; market, technical, organizational and economic -financiero will be released a current situational diagnosis of avocado in Mexico and the target market that will be the United States, which is intended to export the fruit in the best way. The investment project for the production, commercialization and export of avocado of the Booth 8 variety, shows us that we have the necessary information to produce, commercialize and export it. In addition, the target market was specified, where I explain that the pacific part is where there is more avocado consumption and also prefer green varieties such as Booth 8 and Hass. The United States is a large market with more than 300 million inhabitants, almost $45 \%$ consume avocado fruit. The price that will be handled in the local market will be $\$ 15$ per piece and in the target market it will raise its cost to $\$ 35$, so we conclude that it can be said that the Booth 8 avocado production, marketing and export project to the target market is feasible and profitable.

Keywords: Avocado, Food security, Commercialization.

\section{INTRODUCCIÓN}

El problema que limita la producción, comercialización y exportación del aguacate en México y mercados internacionales es la presencia de plagas reglamentadas que impiden su crecimiento en buen estado y movilización.

$\mathrm{El}$ aguacate es atacado por el Barrenador Pequeño del Hueso del Aguacate (Conotrachelus perseae y C. aguacatae), el Barrenador de Ramas del Aguacatero (Copturus aguacatae), el Barrenador Grande del Hueso del Aguacate (Heilipus lauri) y la Palomilla Barrenadora del Hueso (Stenoma catenifer) (Secretaria de economía, 2012) por la presencia de este tipo de plagas es que el cultivo del aguacatero está regulado y su movilización está sujeta a un estricto control, con base a la norma oficial mexicana NOM-066-FITO2002,"Especificaciones para el manejo fitosanitario y movilización del aguacate". Por tal motivo, en coordinación con la Secretaría de Agricultura, Ganadería, Desarrollo Rural, Pesca y Alimentación, SAGARPA y la Secretaría de Desarrollo Agropecuario SDA, implementa la Campaña

Contra Plagas Reglamentadas del Aguacatero con el objeto de apoyar la competitividad del sector agrícola (SAGARPA 2011). El objetivo principal de la investigación es: Demostrar la factibilidad técnica, económica y financiera de un proyecto de inversión de aguacate variedad Booth 8 , cultivado en la zona norte del estado de Veracruz, con fines de exportación. México es el centro de origen del aguacate (Persea americana Mill.). La evidencia más antigua del consumo de esta fruta data de 10,000 años A. C. y fue encontrada en una cueva localizada en Coxcatlán, Puebla. El origen del aguacate tuvo lugar en las partes altas del centro y este de México, y partes altas de Guatemala. En el aguacate existen razas, tres de ellas son más comunes y fueron donde se dio inicio este cultivo a continuación se describirá las tres razas de aguacate más comunes: Generalmente, se identifican tres razas de aguacate: mexicana, guatemalteca y antillana. Uno de los cultivos más significativos para México es el aguacate, originario de México y con gran aceptación a nivel mundial. En el primer trimestre de 2014, la exportación de aguacate aumentó en un $29 \%$ con respecto al mismo período de 2013. Dichas exportaciones suponen un valor de 794.446.000 dólares entre enero y junio de 2014, con un volumen estimado en 353.000 toneladas. El destino de las exportaciones fueron 21 mercados distintos: Estados Unidos, Japón, Canadá, Costa Rica, El Salvador, Honduras, Francia, Guatemala, España, China, Países Bajos, Hong Kong, Reino Unido, Alemania, Singapur y Bélgica, siendo los más prominentes (FND, 2014). El aguacate es un producto de gran 
importancia en la agricultura y en la dieta mexicana. Cuenta con más de 150 mil hectáreas plantadas, menos del $1 \%$ de la superficie cultivable nacional, pero aporta cerca del $3.4 \%$ del valor de la producción agrícola total. La producción de aguacate en 2012 alcanzó 1.3 millones de toneladas y para 2013 se cuenta con una cifra preliminar de 1.5 millones (FND, 2014). Nuestro país es el principal productor mundial, aquí se produce el $30 \%$ de la oferta. Las exportaciones alcanzan en el 2013 cerca de 600 mil toneladas con un valor de más de mil mdd, principalmente dirigidas a EEUU (80\%), Japón (9\%) y Canadá (5\%) (FND, 2014). El mercado estadounidense es el principal consumidor de aguacate Mexicano con 80 por ciento de las exportaciones. El 65 por ciento del fruto que se consume en ese país proviene de México, un 23 por ciento de California, un 7 por ciento de Chile y 5 por ciento de Perú. En 10 años las exportaciones a Estados Unidos crecieron mil 200 por ciento, que equivalen a un crecimiento anual promedio de 30 por ciento. Las ventas a ese país aumentaron poco más del doble en apenas cinco años, pero también muestran un avance cercano en las exportaciones hacia Japón y Canadá, que le siguen en importancia. No obstante, este producto tiene cada vez más demanda en países como Costa Rica, El Salvador, Francia, China, España, Alemania, Reino Unido, Singapur y Rusia, por mencionar algunos, situación que muestra la firmeza de esta industria (FND, 2014).

Este estudio de mercado es el punto de partida para el desarrollo de los capítulos posteriores, como son los estudios técnico, organizacional y financiero, es por ello que se inicia con el desarrollo del estudio de mercado que analiza al aguacate booth 8 , se compone con la identificación de objetivos, descripción del producto análisis de la demanda y oferta, tamaño del mercado para el producto, comercialización, estrategias de comercialización y programa de puesto de ventas. Los objetivos del estudio de mercado son 1). Identificar la demanda existe en el mercado meta. 2). Cuantificar oferta recomendada para exportar. 3). Precisar el mercado meta para nuestro producto. 4). Programa de ventas alineado a la demanda y fenología del cultivo. Los consumidores de EE.UU adquieren productos saludables con un alto valor nutritivo y proteico, que les permitan tener bienestar y tranquilidad en la vida diaria (Procolombia, 2016). El perfil del consumidor de Estados Unidos se caracteriza principalmente por:

-valora la comodidad ante todo y por eso compra cosas que no le compliquen la vida.

-Le preocupa la seguridad, el medio ambiente y la vida sana. Por ello en su alimentación utiliza productos naturales.

- Adquieren productos que no contaminen, que sean ciento por ciento naturales.

- El consumidor de los Estados Unidos se interesa principalmente pro productos frescos (Procolombia, 2016). El empaque generalmente representa un alto porcentaje del costo del producto empacado, lo cual obliga a una selección cuidadosa del empaque y sus materiales.

Una vez considerados los elementos de preparación para la transportación del fruto, es recomendable el uso de camiones refrigerados, pues las fluctuaciones de temperatura provocan la condensación de agua sobre la cáscara de la fruta y esto favorece el deterioro patológico del aguacate y le resta vida comercial (SAGARPA, 2014). Los estadounidenses invierten una cantidad considerable de tiempo y dinero en supermercados. Según el Food Marketing Institute, el comprador promedio americano visita un supermercado alrededor de 88 veces al año, e invierte más de $\$ 6000$ en comida (El planeta.com, 2014). México se ha convertido en un productor internacional monstruo de aguacate al cerrar el 2014 con una cosecha de 1 millón 100,000 toneladas de fruto, monto que representa más de $50 \%$ de la producción mundial que oscilo en 2 millones 100,000 toneladas (El economista, 2014). Nuestro país es el principal productor, consumidor y exportador de aguacate 
en el mundo, superando a países como Chile, España, Israel y Estados Unidos en cuanto a exportaciones (Empacadora de aguacate Avoperla, 2015)

México es líder mundial en exportaciones de aguacate con el 50,3\% por ciento del comercio mundial, seguido por Chile, con el 19 por ciento; España, 13 por ciento, e Israel, doce por ciento. Los principales importadores de aguacate son: o Estados Unidos (78.623 ton), o Francia (105.084 ton) y o Japón (14.070 ton).

El precio en el mercado meta será obviamente mayor, ya que se considera todos los costos que se le da al proceso del aguacate, del momento de la cosecha hasta el puerto destino. El precio en el mercado meta será de $\$ 35$ en pro producto, se manejara sola una presentación de envió del producto, el envase que utilizaremos será un alveolo de material polipropileno con cavidad de 11 aguacates por envase.

La empresa estima producir en el primer año de producción 23,250.00 toneladas con la capacidad del huerto al $25 \%$, el segundo año d producción se estima producir 52,500.00 toneladas con la huerta a su capacidad del $50 \%$ para el tercer año en producción se producirá $78,750.00$ toneladas, con un $75 \%$ de capacidad del huerto para el cuarto año se estima producir un total de 105,000.00 toneladas con el 100\% de capacidad del huerto. El sistema de comercialización Internacional del aguacate está compuesto por un flujo del producto en el cual se involucran una serie de empresas e instituciones mediante la prestación de servicios de transporte, almacenamiento, empaque, distribución y publicidad, entre otras; con el propósito de que el aguacate vaya desde la huerta hasta la mesa del consumidor final. La fuerte demanda de aguacate en EE, UU crece anualmente, cada año es más grande la demanda de este fruto, la variedad hass es la principal variedad que se exporta y comercializa en Estados Unidos, para problema de esta variedad su fecha de corte es en los meses de mayo-julio, en estas fechas es donde hay abundante oferta del producto y su peso oscila entre los 190g$230 \mathrm{~g}$.

La variedad BOOTH8 tiene un peso promedio entre los $900 \mathrm{~g}-1.200 \mathrm{~kg}$. y su fecha de corte es en los meses de noviembre- enero; y el sabor de la variedad BOOTH8 comparando con la variedad HASS, es más cremoso si se consume en su punto exacto ya que contiene más pulpa y más aceite.

En conclusión las tres principales ventajas de la variedad BOOTH8 es el tamaño, el sabor, y la fecha de corte. De acuerdo al estudio de mercado si existe demanda del aguacate de la variedad Booth 8 , el estudio que se realizó arrojo que la zona del pacifico en Estados Unidos es donde más se consume y además son los aguacates de raza verde que tiene preferencia en esa zona, también se sabe en a final de año las importaciones bajan, ya que en México la temporada de aguacate Hass está en desarrollo y es ahí donde existe mucho futuro para la variedad Booth 8 ya que esta variedad se empieza a cosechar en el mes de Noviembre y su fecha de corte acaba en el mes de enero. Para que nuestro producto sea de calidad es muy importante contar con las certificaciones mexicanas, en nuestro país son las Buenas Prácticas Agrícolas, que consta en certificar que los alimentos no causara daño al consumidor. La dependencia de gobierno que apoya para obtener esta certificación es el Servicio Nacional de Sanidad, Inocuidad y Calidad Agroalimentaria (SENASICA). Para enviarlo es necesario la utilización del envase de alveolos se asegura la protección del fruto durante el traslado del lugar del almacén hasta el punto final con el cliente. Debido a que el envase se encuentra fabricado de polipropileno, nos facilita la reutilización de este ya que puede ser esterilizado a temperaturas de 140 grados centígrados, además que el propio material cuenta con una propiedad la cual resiste la penetración de microorganismos (Packagingci, 2016). La exportación del producto de aguacates BOOTH8 "aguacates Ixca-Ver" se realizara a través del puerto de Tuxpan. Se encuentra localizada a 14.8 kilómetros al sur de las escolleras del Rio 
Tuxpan. C.P. 92884 Tuxpan de Rodríguez Cano, Veracruz (México). Empresa naviera a contratar es la empresa MELFI MARINE CORPORATION S.A. la cual ofrece un cuidado profesional para el producto, asegurándose que se mantenga en buenas condiciones y es una de las navieras más grandes con servicios. el tercer año será el primero en producción del huerto y es ahí donde será el punto de partida, el primer año en producción que es el tercer año del cultivo la capacidad del huerto se estima que sea de un $25 \%$ para el cuarto año seria de una $50 \%$ para el quinto año de un $75 \%$ para el año seis se estará alcanzando el $100 \%$ del huerto y para el año 7, 8,9 y 10 el huerto estará en capacidad plena.

\section{CONCLUSIONES}

Después de haber concluido el proyecto de inversión para la producción, comercialización y exportación de aguacate de la variedad Booth 8, en el municipio de Ixcatepec, Veracruz, podemos decir que se cuenta con la información necesaria para producir, comercializar y exportar aguacate Booth 8. El proyecto tuvo la finalidad de que sea rentable y dio un aporte significativo al sector productivo de la zona norte de Veracruz Ixcatepec Ver. El estudio económico financiero, contempla un financiamiento otorgado por la FND; En el sexto año del huerto es donde se alcanza la entrada en producción del 100\% de la superficie, en total el costo total de producción al sexto año será de $\$ 2,166,727.81$, el volumen de producción será de 105, 000. 00 toneladas por temporadas y nuestros ingresos será de $\$ 3,675$, 000. 00. Con este análisis se comprueba que el proyecto es rentable que incluso en el segundo año de producción, son mayores los ingresos que los egresos.

Es por eso que este proyecto de inversión se puede replicar en la zona norte del estado de Veracruz.

\section{LITERATURA CITADA}

Baiza. V. H. A. (2003). Guía técnica del cultivo de aguacate. P.69. Guatemala. Editorial Maya. P. 123.

Barrientos Priego, A. F., y López-López, L. (2000). Historia y genética del aguacate. Téliz, D. y Mora, A. (Comps.). El aguacate y su manejo integrado. $2^{\mathrm{a}}$ (Ed.) Ediciones Mundi-Prensa. D.F. México. P. 22-62.

Barrientos-Priego, A.F., M.W. Borys, E. EscamillaPrado, A. Ben-Ya'acov, E. de la Cruz-Torres, y L. López-López. 1992. Study of avocado germplasm resources, 1988-1990. IV. Findings in the Mexican Gulf region. Proc. Of Second World Avocado. P. 551-558.

Centeno. G. (2015). El mercado estadounidense para aguacate fresco convencional. Estados Unidos. P. 16.

Cornell. C. y Stanley .B. (2007). Economía, principios, problemas y políticas. 14ta edición. vol.14. Colombia: Nomos S.A. P.480.

Días Tipan, M. M. (2013). Producción, comercialización y rentabilidad de la naranja (Citrus aurantium) y su relación con la economía del cantón y la maná y su zona de influencia .Tesis ingeniería. Universidad Técnica de Cotopaxi. P. 83.

Financiera Nacional de Desarrollo Agropecuario, Rural, Forestal, y Pesquero. (2014). Panorama del aguacate. FND. P. 33.

Keat. P. (2007). Economía de empresa. Cuarta edición. Vol.4. México: Person educación. P.760.

Krugman. P. y Wells. R. (2007). Introducción a la economía: microeconomía. Tercera edición. Vol.3. Barcelona, España: Reverte S.A. P.123.

Línea Agrícola. (2012). Manejo fitosanitario del cultivo de aguacate has. Colombia. P.75.

Martínez, L. A. (2013). Alimentos Procesados. Secretaría de Economía. México: PROMEXICO. P. 1

Martínez. F. J. R. (2014). Plan Municipal de Desarrollo 2014-2017.Presidencia municipal de Ixcatepec, Veracruz. México. P. 33.

Miranda, M. J. (2005). Gestión de proyectos. Identificación - formulación - evaluación: Financiera - económica - social - ambiental. [Versión electrónica]. Colombia: MM Editores. P. 58. 
Velásquez. J. S. (2000) Recomendaciones técnicas para el cultivo d aguacate. P. 35.

Oficina de información diplomática. (2015). Ficha país Estados Unidos. Estados Unidos. P. 2.

Organización de las Naciones Unidas para la Alimentación y la Agricultura. P.1.

Secretaria de Agricultura, Ganadería, Desarrollo Rural, Pesca y Alimentación. Monografía de cultivos. México. SAGARPA, (2011). P.1.
Secretaria de Desarrollo Rural del Estado de Puebla. (2007). Catálogo de propiedades nutrimentales, nutraceuticas y medicinales del aguacate. Coordinación general de cadenas productivas. Puebla. SDR. P.11AlarcónChaires, P. 2010. Etnoecología de los indígenas $\mathrm{P}^{\prime}$ urhèpecha. Una guía para la aproximación de la apropiación de la naturaleza. UNAM, México.

Copyright (c) 2017 Rubén de la Paz Mendo Muñoz, Benjam in Cruz Ramirez,

Miguel Ángel G onzález Salas y Jor dán Gutiérrez Vivanco

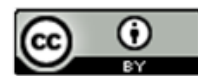

Este tex to está protegido por una licencia licencia Creative Cormmons 4.0

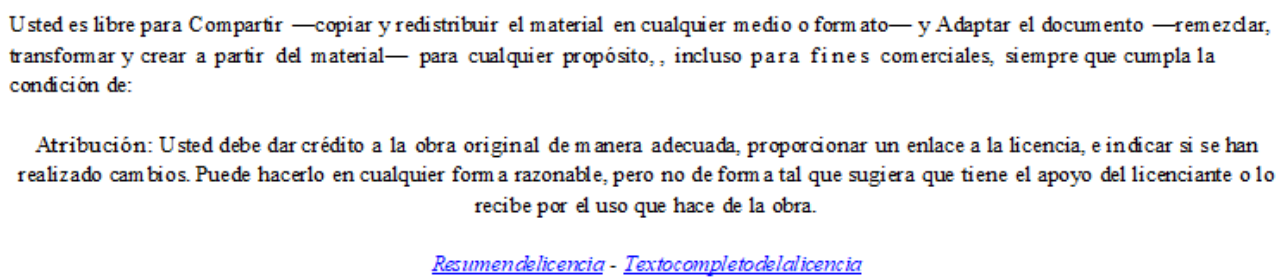

Resumenclelicencia - Textocompletocielalicencia 